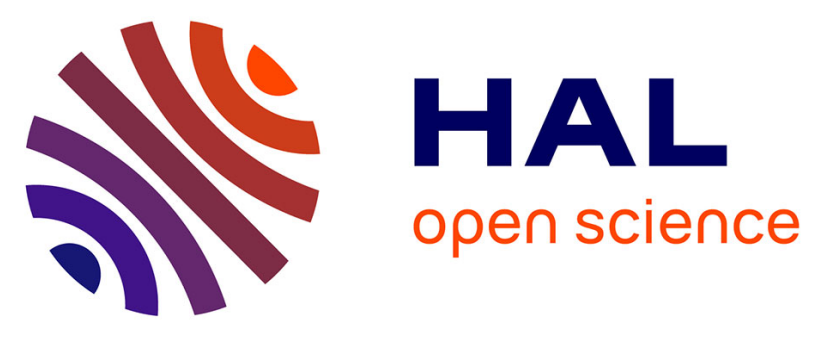

\title{
Postprandial Triglyceride-Rich Lipoproteins from Type 2 Diabetic Women Stimulate Platelet Activation Regardless of the Fat Source in the Meal
}

Marie Michèle Boulet, David Cheillan, Mathilde Di Filippo, Taïssia

Lelekov-boissard, Charline Buisson, Stéphanie Lambert-porcheron, Julie-anne Nazare, Jessica Tressou, Marie-Caroline Michalski, Catherine Calzada, et al.

\section{To cite this version:}

Marie Michèle Boulet, David Cheillan, Mathilde Di Filippo, Taïssia Lelekov-boissard, Charline Buisson, et al.. Postprandial Triglyceride-Rich Lipoproteins from Type 2 Diabetic Women Stimulate Platelet Activation Regardless of the Fat Source in the Meal. Molecular Nutrition and Food Research, 2020, 64 (19), pp.2000694. 10.1002/mnfr.202000694 . hal-03015175

\author{
HAL Id: hal-03015175 \\ https://hal.science/hal-03015175
}

Submitted on 7 Dec 2020

HAL is a multi-disciplinary open access archive for the deposit and dissemination of scientific research documents, whether they are published or not. The documents may come from teaching and research institutions in France or abroad, or from public or private research centers.
L'archive ouverte pluridisciplinaire HAL, est destinée au dépôt et à la diffusion de documents scientifiques de niveau recherche, publiés ou non, émanant des établissements d'enseignement et de recherche français ou étrangers, des laboratoires publics ou privés. 


\section{$1 \quad$ Postprandial triglyceride-rich lipoproteins from type 2 diabetic}

\section{2 women stimulate platelet activation regardless of the fat source in}

\section{the meal}

4 Marie Michèle Boulet ${ }^{1}$, David Cheillan ${ }^{1,2}$, Mathilde Di Filippo ${ }^{1,2}$, Taïssia Lelekov-Boissard ${ }^{3}$,

5 Charline Buisson ${ }^{1}$, Stéphanie Lambert-Porcheron ${ }^{4}$, Julie-Anne Nazare ${ }^{4}$, Jessica Tressou ${ }^{5,6}$,

6 Marie-Caroline Michalski ${ }^{1}$, Catherine Calzada ${ }^{1 *}$, Philippe Moulin $^{1,3^{*}}$

7

8

9

10

${ }^{1}$ Univ-Lyon, CarMeN Laboratory, Inserm U1060, INRAE UMR 1397, INSA Lyon, Université Claude Bernard Lyon 1, IMBL, Villeurbanne, France.

${ }^{2}$ Laboratoire de Biochimie et de Biologie Moléculaire Grand Est, Centre de Biologie et de Pathologie Est, Hospices Civils de Lyon, Bron, France.

${ }^{3}$ Fédération d'endocrinologie, Maladies Métaboliques, Diabète et Nutrition, Hôpital Louis Pradel, Hospices Civils de Lyon, Bron, France.

${ }^{4}$ Centre de Recherche en Nutrition Humaine Rhône-Alpes, Univ-Lyon, CarMeN Laboratory, Université Claude Bernard Lyon1, Hospices Civils de Lyon, CENS, FCRIN/FORCE Network, Pierre Bénite, France.

${ }^{5}$ UMR MIA-Paris, AgroParisTech, INRAE, Paris-Saclay University, Paris, France

${ }^{6}$ Graduate School of Agricultural and Life Sciences, The University of Tokyo, Tokyo, Japan

*CC and PM contributed equally

\section{Address for correspondence and reprint requests:}

Catherine Calzada, Ph.D. 
24 Inserm U1060 - Université Claude Bernard Lyon 1 - INRAE UMR1397 - INSA-Lyon

25 CarMeN Laboratory

26 Bâtiment CENS-ELI 2D

27 Hôpital Lyon Sud Secteur 2

28165 chemin du Grand Revoyet

2969310 Pierre-Bénite, France

30

31 Abbreviations list: AA: arachidonic acid, CM: chylomicrons, HCS: hazelnut cocoa spread,

FA: fatty acids, LPL: lipoprotein lipase, PP: postprandial, PPP: platelet-poor plasma, PRP: platelet-rich plasma, SFA: saturated fatty acid, TC: total cholesterol, TG: triglycerides, 34 TGRL: triglyceride-rich lipoproteins, $\mathrm{TxB}_{2}$ : thromboxane $\mathrm{B}_{2}$

35

Keywords: fatty acids, platelets, postprandial, triglyceride-rich lipoproteins, type 2 diabetes 


\section{ABSTRACT}

Scope. The aim of this study was to examine whether postprandial (PP) triglyceride-rich lipoproteins (TGRL) secreted after a moderate fat intake would activate platelets differently according to their fatty acid (FA) composition.

Methods and results. In a parallel single-blind randomized trial, 30 women with type 2 diabetes were assigned to a breakfast containing $20 \mathrm{~g}$ lipids from butter $v s$ hazelnut cocoa spread rich in palm oil. Blood samples were collected at fasting and $4 \mathrm{~h}$ PP. FA composition of fasting and PP TGRL and their effects on the activation of platelets from healthy blood donors were assessed. Both breakfasts similarly increased plasma ApoB-48, plasma and TGRL triglycerides $(\mathrm{p}<0.05)$. TGRL mean diameter increased after both breakfasts and was greater after the butter breakfast. Both breakfasts were rich in palmitic acid, and the HCS breakfast contained $45 \%$ oleic acid. TGRL FA composition reflected the dietary FA composition. Pre-incubation of platelets with fasting and PP TGRL increased collagenstimulated aggregation ( $\mathrm{p}<0.01$ vs control). Fasting and PP TGRL similarly increased agonistinduced thromboxane $\mathrm{B}_{2}$ concentrations, and this effect was concentration-dependent for PP TGRL.

Conclusion. PP TGRL from type 2 diabetic women after a palm-oil spread vs butter-based mixed meal induce similar acute in vitro platelet activation. 


\section{INTRODUCTION}

Type 2 diabetic (T2D) patients have a higher risk of cardiovascular diseases, including ischemic coronary artery events, atherosclerosis and atherothrombosis [1]. Epidemiologic studies also showed that women with T2D exhibit a worse cardiovascular risk profile [2] and have greater increase in cardiovascular morbidity and mortality compared to men [3]. Platelets from T2D individuals have been shown to be hyperactive, thus more easily prone to agonist-triggered aggregation in the event of plaque rupture [4]. Moreover, it is well documented that increased concentration of plasma triglycerides (TG), carried in the bloodstream by "triglyceride-rich lipoproteins" (TGRL) that are chylomicrons (CM), VLDL and their remnants, is an important risk factor in T2D [5]. Our group previously showed that large TGRL from fasting T2D patients enhanced in vitro platelet activation and aggregation [6].

It is now well acknowledged that postprandial (PP) TG concentrations are involved in cardiovascular risk. The concept of the atherogenicity of CM and VLDL was first introduced by Zilversmit 40 years ago [7]. Non-fasting TG levels were subsequently associated with the incidence of various cardiovascular events [8-12], independently of other risk factors [13, 14]. TGRL remnants are likely to be the main contributors of an enhanced atherosclerosis development $[14,15]$. However, regarding atherothrombosis, the contribution of TGRL is not clearly established. The few studies on this topic were performed mostly in non-diabetic hyperlipidemic and normolipidemic individuals and had major differences in methodology. They showed that CM from both groups can have an inhibitory effect on platelet functions [16], while VLDL from healthy subjects [17] and remnants from healthy and T2D individuals as well as patients with lipoprotein lipase (LPL) deficiency [18] would have an enhancing effect. No study had been performed on the potential implication of PP TGRL from T2D 
82

patients in atherothrombosis, and it is relevant to study, especially in this population at higher risk for cardiovascular complications.

Because the CM fraction of TGRL in the PP state is mainly constituted of dietary TG, the fatty acid (FA) composition of the ingested lipid influences their fatty acid composition in healthy individuals [19]. It is also known that there are exchanges of FA from plasma and lipoproteins to phosphatidylcholine in platelet membranes [20, 21]. Chain length of the saturated fatty acids (SFA) can also impact platelet aggregation. Studies in humans suggest deleterious stimulating effects of dietary long chain SFA on platelet function [22-24]. Also, it was shown that the PP state was associated with increased platelet activation in T2D patients following the consumption of a carbohydrate-rich meal and related to PP insulin [25, 26]. However the specific effect of TGRL secreted following the consumption of fats with different SFA composition on platelet aggregation has never been investigated in T2D women.

In this context, we hypothesized that PP TGRL from T2D women would have a modified FA composition following a realistic fat intake via a mixed meal from different food sources (dairy vs vegetal) and that this would change their effect on platelet activation and aggregation. 


\section{EXPERIMENTAL SECTION}

Study participants

Thirty T2D women were recruited at the Department of Endocrinology and Metabolic Diseases, Cardiovascular Hospital, Lyon Bron (France) and randomized in one of the two groups. To be included, the subjects required to be women aged between 50 and 80 years old, with a post-menopausal status, a stable weight and a BMI $\geq 27$ and $\leq 40 \mathrm{~kg} / \mathrm{m}^{2}$, an $\mathrm{HbA}_{1 \mathrm{c}} \geq 6,5$ and $\leq 10 \% \quad(\geq 48$ and $\leq 86 \mathrm{mmol} / \mathrm{mol})$ and no history of cardiovascular events or major hypertriglyceridemia. The anti-diabetic and lipid lowering medication was not discontinued for ethical reasons: 28 (93\%) had metformin, $18(60 \%)$ had sulfonylurea, $11(37 \%)$ had glucagon-like peptide 1 analog, 8 (27\%) had gliptin and 1 (3\%) had Acarbose; none was treated with insulin injection. In addition, $17(57 \%)$ had statins and $1(0.03 \%)$ had a combined therapy of statins and ezetimibe. Table 1 shows study participants characteristics. They were postmenopausal women with grade 1 obesity (mean BMI of $34.1 \pm 1.0$ ), and long duration of T2D (12.9 \pm 1.4 years). They had low serum LDL cholesterol due to statin treatment and none was treated with fibrates. They had a mild increase in serum TG concentration $(33.3 \%$ had plasma TG above $2 \mathrm{mmol} / \mathrm{L})$.

\section{Study design}

The study is a randomized interventional parallel trial entitled The Composition of Triglyceride Rich Lipoproteins and Platelet Activation in Type 2 Diabetes (COMPLETE, clinical trial registration number at ClinicalTrials.gov: NCT03561571). The trial was approved by the Scientific Ethics Committee Ile de France VII and conducted in accordance with the principles of the Helsinki declaration. Informed written consent was obtained from all subjects. The subjects performed the trial from October 2018 to May 2019. A minimum sample size of $10 \mathrm{~T} 2 \mathrm{D}$ women per group was necessary to detect a $30 \%$ difference in $\mathrm{TxB}_{2}$ 
concentrations with an alpha level of 0.05 and $80 \%$ power, 15 women were recruited in each group to increase the statistical power. The primary outcome was the difference in $\mathrm{TxB}_{2}$ concentrations in platelets incubated with fasting and PP TGRL. Secondary outcomes were the difference in the aggregation of platelets incubated with fasting and PP TGRL, the difference in $\mathrm{TxB}_{2}$ concentrations in platelets incubated with TGRL collected after the consumption of a fat of different FA composition; and the difference in FA composition between fasting and PP TGRL from each group.

The interventions were randomized according to a block randomization performed by a biostatistician associated to the study using the R software (The R Foundation, Miami, FL). The block randomization ( 7 blocks of 4 and 1 block of 2 patients) was generated so that both treatments could be tested on platelets from the same donor in each block. The outcome assessors were blinded to the group assignment of the patients. After a 12 hour overnight fast, the first blood draw was made and the subjects were asked to eat the whole test breakfast according to their randomization group. A second blood sample was collected 4 hours after the ingestion of the breakfast. Blood sampling and test breakfast administration were done as part of the annual T2D follow-up at the outpatient clinic.

\section{Test breakfasts composition}

Both experimental breakfasts contained $20 \mathrm{~g}$ of fats, one containing a majority of vegetal oil (mostly refined palm oil) within a commercial hazelnut-cocoa spread (HCS) and the other with butter. The FA composition of both fats was assessed by gas chromatography from 30 mg of butter and $70 \mathrm{mg}$ of HCS with the same method as for the TGRL. Lipids were extracted with chloroform/methanol (2:1) according to the Folch method [27]. The HCS mostly contained palmitic (16:0) and oleic (18:1 n-9) acids. Butter contained a broader range of SFA 
(Table 2). Both breakfasts were designed to be similar in carbohydrates, proteins and caloric contents. The butter breakfast was composed of plain crackers $(30 \mathrm{~g})$, commercial butter (22.5g, $82 \%$ fat), jam (30g), orange juice $(200 \mathrm{ml})$ and plain coffee or tea $(200 \mathrm{ml})$ for a total of 440 calories, with $51.7 \%, 40.9 \%$ and $4.5 \%$ of energy as carbohydrates $(56.9 \mathrm{~g})$, lipids $(20 \mathrm{~g})$ and proteins $(5 \mathrm{~g})$, respectively. The HCS breakfast contained plain crackers $(30 \mathrm{~g})$, commercial HCS $(60 \mathrm{~g})$ and plain coffee or tea $(200 \mathrm{ml})$ providing 439 calories with $51.1 \%$, $40.9 \%$ and $6.5 \%$ of energy as carbohydrates $(56.1 \mathrm{~g})$, lipids $(20 \mathrm{~g})$ and proteins $(7.1 \mathrm{~g})$, respectively. All subjects were given a maximum of 20 minutes to eat the breakfast.

\section{Plasma biochemistry}

Blood was collected into EDTA tubes and plasma was immediately isolated by centrifugation $\left(1500 \times \mathrm{g}, 10 \mathrm{~min}\right.$, at $\left.4{ }^{\circ} \mathrm{C}\right)$ and stored in aliquots at $-20{ }^{\circ} \mathrm{C}$. Measurement of ApoB-48 concentrations was performed by ELISA according to the manufacturer's instruction (SHIBAYAGI, Shibukawa, Gunma Prefecture, Japan). Total (TC), HDL cholesterol (HDL-c), TG, total ApoB and glucose were enzymatically determined using commercial kits (ABBOTT Diagnostics) on an Architect C16000 autoanalyser (ABBOTT Diagnostics, Illinois, USA). LDL cholesterol was calculated using Friedewald equation $($ LDL-c $=$ TC - HDL-c $\left.0.45^{*} \mathrm{TG}\right) . \mathrm{HbA}_{1 \mathrm{c}}$ was determined by capillary electrophoresis using a Sebia Capillarys 3 (Sebia). Insulin was determined by immunoassay on an Architect I2000 autoanalyser. ApoB100 was calculated by subtraction of ApoB-48 from total ApoB.

\section{Isolation and characterization of TGRL fractions}

In order to collect TGRL fractions, $250 \mu \mathrm{L}$ of plasma was carefully deposited under a layer of distilled water $(850 \mu \mathrm{L})$ followed by ultracentrifugation at $9777 \times \mathrm{g}$ for 1 hour at $12^{\circ} \mathrm{C}$ using a 
173 Beckman Coulter Optima TLX ultracentrifuge and a Beckman TLA 100.2 rotor. Top $100 \mu \mathrm{L}$ 174 of the floating layer corresponding to TGRL with density $<1.000 \mathrm{~g} / \mathrm{ml}$ was collected and 175 stored at $4{ }^{\circ} \mathrm{C}$ for no longer than 2 days or stored at $-20^{\circ} \mathrm{C}$ for lipid analysis. Protein 176 concentration of the fractions was measured using a modified Lowry method [28]. TG 177 concentrations were obtained on an Architect C16000 autoanalyser. The hydrodynamic 178 diameter of TGRL was measured by dynamic light scattering at $25^{\circ} \mathrm{C}$ with a ZetaSizer NanoS 179 (Malvern, UK) using 1.0658 as viscosity and 1.445 as refractive index and 1.330 as refractive 180 index of the aqueous phase [29].

TGRL fatty acid composition by gas chromatography

Total lipids were extracted twice from TGRL $\left(200 \mu \mathrm{L}+300 \mu \mathrm{L} \mathrm{H}_{2} \mathrm{O}\right)$ with ethanol:chloroform (1:3:6, respectively) after addition of 1,2,3-triheptadecanoyl-sn-glycerol as internal standard

185

\section{Platelet isolation}

Venous blood was obtained from the local blood bank (Etablissement Français du Sang) on citrate-phosphate-dextrose anticoagulant $(19.6 \mathrm{mmol} / \mathrm{L}$ citric acid, $89.4 \mathrm{mmol} / \mathrm{L}$ sodium citrate, $16.1 \mathrm{mmol} / \mathrm{L} \mathrm{NaH}_{2} \mathrm{PO}_{4}, 128.7 \mathrm{mmol} / \mathrm{L}$ dextrose; $\mathrm{pH}$ 5.6) from healthy volunteers who had not ingested any aspirin or anti-inflammatory drugs in the previous 10 days. Platelet-rich plasma (PRP) was prepared by centrifugation of the blood at $200 \times \mathrm{g}$ for 17 minutes at $20^{\circ} \mathrm{C}$. 
197 PRP was collected, acidified to $\mathrm{pH} 6.4$ with $0.15 \mathrm{M}$ citric acid and centrifuged at $900 \times \mathrm{g}$ for 19812 minutes at $20^{\circ} \mathrm{C}$. Platelet-poor plasma (PPP) was removed and platelets were suspended in 199 a Tyrode-HEPES buffer ( $\mathrm{pH}$ 7.35) [30]. The mean concentration for all platelet suspensions was $239 \pm 24 \mathrm{G} / \mathrm{L}$ (min: $141 \mathrm{G} / \mathrm{L}$, max: $354 \mathrm{G} / \mathrm{L}$ ).

\section{Platelet aggregation experiments}

203

204

Aggregation was measured in isolated platelets in a Chrono-log dual-channel aggregometer (Coulter, Margency, France) according to the method of Born [31]. Platelet suspensions were preincubated for 5 minutes at $37^{\circ} \mathrm{C}$ in the presence or absence of different preparations of TGRL (25 $\mu$ g proteins/mL) and then stimulated with subthreshold concentrations of collagen $(0.13 \pm 0.02 \mu \mathrm{g} / \mathrm{mL})$ (type I from equine tendons, Diagnostica Stago, Asnières sur Seine, France) with continuous stirring at $1000 \mathrm{rpm}$. The subthreshold concentrations of collagen were defined as the concentrations of collagen that induced approximately less than a $10-20 \%$ increase in light transmission. The extent of platelet aggregation was expressed as percentage of change in light transmission 4 minutes after the addition of collagen [32].

\section{Platelet thromboxane $B_{2}$ measurement}

Freshly isolated platelet suspensions were incubated at $37^{\circ} \mathrm{C}$ for 30 minutes in the absence or presence of TGRL ( $25 \mu \mathrm{g}$ proteins/mL) while being slowly agitated. Platelets were incubated at $37^{\circ} \mathrm{C}$ for an hour with non aggregating concentrations of collagen $(0.5 \mu \mathrm{g} / \mathrm{mL})$ or thrombin $(0.05 \mathrm{U} / \mathrm{mL})$ while being slowly agitated before being immediately stored at $-80^{\circ} \mathrm{C}$ until $\mathrm{TxB}_{2}$ measurement. Following 3 cycles of thawing and freezing of samples for platelets lysis, $\mathrm{TxB}_{2}$ concentrations were determined by enzyme immunoassays according to the manufacturer's recommendations (Enzo Life Sciences Inc., Villeurbanne, France). Platelet proteins 
221 concentrations were measured using the Bradford method [33] in order to express $\mathrm{TxB}_{2}$

222 results.

223

224 Statistical analysis

225 Data were expressed as means \pm SEM. Normality of data distribution was assessed using 226 d'Agostino-Pearson normality test. Paired Student's t-test or Wilcoxon test was used to assess 227 differences between platelets incubated with TGRL and stimulated with an agonist compared 228 to platelets stimulated with an agonist. Differences between platelets stimulated with an 229 agonist compared to platelets alone were performed to ensure platelet functionality in 230 response to agonists using Student's t-test or Wilcoxon test as well. Differences between 231 T240 and T0 in each group were assessed using paired Student's $t$-test or Wilcoxon test and 232 differences between the two groups for the means of variation deltas (T240-T0) were 233 determined using unpaired Student's $t$-test or Mann-Whitney test. Differences were considered significant at $\mathrm{p}<0.05$ ( 2 sided test). All statistical analyses were performed using GraphPad Prism 8.0 (Graphpad Software, San Diego, CA). 


\section{RESULTS}

\section{Postprandial modification of plasma and triglyceride-rich lipoproteins}

At the plasma level, consumption of both breakfasts increased ApoB-48 (HCS: +78\%, Butter: $+67 \%, \mathrm{p}<0.001$ for both) and TG (HCS: $+33 \%$, Butter: $+30 \%, \mathrm{p} \leq 0.0001$ for both) concentrations similarly (Figure 1A and B) at 4 hours after 20g fat meal. TGRL isolated from plasma of fasting T2D patients had a mean diameter of $146 \pm 5 \mathrm{~nm}$ and a mean TG and total FA concentrations of $0.07 \pm 0.01 \mathrm{mmol} / \mathrm{L}$ and $512 \pm 6.5 \mathrm{nmol} / \mathrm{mg}$ protein, respectively. TGRL collected after the breakfasts had an increased diameter, TG and FA content $171 \pm 4 \mathrm{~nm}$, $0.12 \pm 0.02 \mathrm{mmol} / \mathrm{L}$ and $907 \pm 14 \mathrm{nmol} / \mathrm{mg}$ protein, respectively. TGRL mean concentration of TG was significantly increased in the PP state. As shown in Figure 2A, there was a 200\% and $71 \%$ increase in TGRL-TG after the HCS and butter breakfasts, respectively ( $p<0.01$ for both groups). The type of fat does not impact TGRL-TG concentrations in PP plasma. Following the HCS breakfast, mean TGRL diameter was $10 \%$ higher, while it was increased by $23 \%$ after the butter breakfast (Figure 2B) ( $\mathrm{p}<0.01$ for both groups). This increase in diameter after the butter breakfast was significantly higher than after the HCS breakfast (Figure 2B, $\mathrm{p}<0.01)$.

As shown in Table 3, the main FA found in TGRL were palmitic (16:0), oleic (18:1 n-9) and linoleic (18:2 n-6) acids and their molar percents were not different between both groups in the fasting state. Ingestion of the HCS breakfast increased the proportion of oleic acid, the main MUFA, with decreased proportions of SFA and PUFA. After the butter breakfast, molar percentages of palmitic acid, the main SFA, and of medium chain SFA were significantly elevated, except for stearic acid (18:0), and proportions of PUFA were significantly decreased. As shown in Figure 3, there was a significant PP increase in TGRL SFA molar 
percent (T240-T0) following the butter breakfast compared to the HCS one, while the MUFA molar percent increased in TGRL after the HCS breakfast compared to the butter breakfast.

\section{Effect of fasting and postprandial TGRL on platelet aggregation}

As shown in Figure 4, TGRL from fasting T2D patients increased collagen-stimulated platelet aggregation (HCS group: $+62 \%$, butter group: $+86 \%, \mathrm{p}<0.01$ for both) compared with collagen-stimulated platelets alone. When platelets were pre-incubated with TGRL isolated in the PP state, collagen-stimulated aggregation increased similarly in both groups (HCS group: $+86 \%$, butter group: $+129 \%, \mathrm{p}<0.01$ for both). When platelets pre-incubated with PP TGRL are compared to platelets pre-incubated with fasting TGRL, the difference was not significant.

\section{Effect of fasting and postprandial TGRL on platelet thromboxane $B_{2}$ concentration}

Concentrations of $\mathrm{TxB}_{2}$ in collagen and thrombin-stimulated platelets alone or pre-incubated with TGRL from T2D patients before and after the test breakfasts are shown in Figure 5. When platelets were stimulated with non-aggregating concentrations of collagen, TGRL isolated in the fasting state in both groups significantly increased $\mathrm{TxB}_{2}$ concentration similarly (HCS group: $+28 \%$, butter group: $+31 \%, \mathrm{p}<0.05$ for both) compared to collagenstimulated platelets. TGRL isolated after the breakfasts did not further increase platelet $\mathrm{TxB}_{2}$ concentration compared to TGRL collected in the fasting state (HCS group: $+29 \%, \mathrm{p}=0.07$, butter group: $+23 \%, \mathrm{p}=\mathrm{NS}$ vs control, Figure 5A). When platelets were pre-incubated with fasting and PP TGRL and stimulated with thrombin, there was an increase of $\mathrm{TxB}_{2}$ platelet concentration (HCS group at T0: $+54 \%, \mathrm{p}=0.06$, HCS group at T240: $+54 \%, \mathrm{p}=0.02$, butter group at T0: $+34 \%, \mathrm{p}=0.007$, butter group at $\mathrm{T} 240:+55 \%, \mathrm{p}=0.001$ vs control) $($ Figure $5 \mathbf{B})$. 
285 We also tested the effect of a higher concentration of PP TGRL ( $50 \mu \mathrm{g} / \mathrm{mL})$ on platelets, since 286 TGRL concentration is elevated in the PP compared to the fasting state. The incubation of 287 platelets with $50 \mu \mathrm{g} / \mathrm{mL}$ of PP TGRL significantly increased $\mathrm{TxB}_{2}$ concentration compared to $28825 \mu \mathrm{g} / \mathrm{mL}$ PP TGRL and fasting TGRL. This effect was not different between the HCS and 289 butter group (Figure 6). 
292

The association of non-fasting TG concentrations with the risk of ischemic cardiovascular events, independently of traditional risk factors, has highlighted the need of specific studies in order to understand the implication of PP TGRL on platelet function. Our randomized controlled study establishes that TGRL secreted in the PP state have a stimulatory effect on platelet aggregation. This finding extends our previous observation of the enhancing effect of large TGRL from fasting T2D patients on platelet aggregation [6]. This effect of PP TGRL was not dependent on their FA composition.

The concentrations of plasma TG and ApoB-48 increased in T2D patients after the ingestion of both realistic breakfasts in a similar way. This is in accordance with the observations of Sciarrillo et al. who showed that plasma TG levels in lean and obese subjects were increased in a similar manner after a meal containing $61 \%$ of calories $(51-84 \mathrm{~g})$ from different fats (butter, coconut oil, olive oil and canola oil) [34]. Our results support the fact that PP TG metabolism is altered in the T2D population, because a similar lipemic response in healthy individuals would necessitate around 30-40g of lipids [35].

Residual fasting TGRL have a mean diameter of $146 \mathrm{~nm}$, which is much larger than what is reported in healthy individuals. For example, Mekki et al. reported a mean diameter of $\sim 91$ $\mathrm{nm}$ for fasting TGRL in healthy individuals [36]. Interestingly, despite a similar amount of fat (20 g), we observed a greater diameter of PP TGRL 4 hours after the ingestion of the butter breakfast $(183 \mathrm{~nm})$ compared to the PP TGRL after the HCS breakfast $(159 \mathrm{~nm})$, and compared to the mean diameter reported for PP TGRL 4 hours after the consumption of a meal containing $40 \mathrm{~g}$ of butter $(\sim 120 \mathrm{~nm})$. In addition, our study shows that ingestion of a moderate amount of lipids (20g) leads to modification of the FA composition of TGRL 4 hours after the intake in T2D women. FA composition of PP TGRL reflects the main FA 
composition of each fat consumed. TGRL secreted after the HCS breakfast, containing $45 \%$ of oleic acid, were enriched in MUFA (particularly oleic acid, 18:1 n-9). Those collected after the butter breakfast, which contained $79 \%$ of SFA (mainly palmitic acid, 16:0) and were accordingly rich in SFA (53\% of total FA), especially palmitic acid (16:0).

The increased size of TGRL collected after the butter breakfast is intriguing and the difference in FA composition of TGRL after both breakfasts could be due to the positional distribution of fatty acids on the glycerol backbone of the TG molecules. In butter, palmitic acid (16:0) is mostly in the $s n-2$ position, while it is on the external sn- 1 and 3 positions in TG from palm oil [37]. Our results are in line with studies on animal models demonstrating that the FA position at $s n-2$, particularly for palmitic acid (16:0), has a determining effect on its absorption and subsequent incorporation in TG [38, 39]. The FA in the $s n-2$ position of the ingested TG is favorably incorporated in the CM [40] and the presence of palmitic acid in the $s n-2$ position is likely to increase TG absorption [41]. However, in our case the palmitic acid content of both fats was similar and this may have minimized the effect of stereospecificity on TG absorption. Also, although the PP TGRL is significantly higher by $24 \mathrm{~nm}$ after the butter breakfast, it may not be enough of an increase to have a physiological impact on TGRL lipolysis.

Our results on the effect of TGRL from T2D patients on platelets show that PP TGRL significantly stimulate in vitro platelet aggregation. To the best of our knowledge, no study has already investigated the specific effect of PP TGRL from T2D patients on isolated platelets. For example, Aviram et al. tested the effect of different isolated lipoproteins fractions from healthy or hyperlipoproteinemic men after two meals containing $50 \mathrm{~g}$ of fat either rich in SFA (25g) or PUFA (38g) on platelet aggregation. They observed that $3 \mathrm{~h}$ after both meals, CM decreased collagen-stimulated aggregation while VLDL enhanced it in both groups [42]. An original aspect of our study is that we isolated and tested both fractions from 
the fasting and PP state, which are different in composition. Indeed, the presence of large TGrich particles in the basal state suggest that these are remnants of an inadequate $\mathrm{CM}$ and VLDL lipolysis [43], while the largest TGRL fractions isolated in the PP state contain CM and are representative of the heterogeneous mix of particles following a meal. We also show for the first time in vitro that an increase in TGRL, in physiological concentration, in the PP state has an enhancing effect on platelets $\mathrm{TxB}_{2}$ concentration after a meal containing a realistic amount of fat rich in SFA. The increase in platelet activation and aggregation was similar after both fats, even though the TGRL FA composition was modified in PP. It is possible that an acute intervention of one breakfast containing a moderate quantity of lipids is not enough to cause a sufficient change in TGRL composition leading to a significant effect of TGRL FA on TGRL-induced platelet activation. A longer dietary intervention with different lipid amount and sources could induce more pronounced effects. Delgado-Lista et al studied in 20 healthy young men the effects of a chronic 28 days intake of a controlled diet followed by 3 tests meals containing $1 \mathrm{~g} / \mathrm{kg}$ of lipids of different FA composition [44]. A lipidrich meal caused an increase of plasma tissue-type plasminogen activator, $\mathrm{d}$-dimers and $\mathrm{TxB}_{2}$ in the PP irrespective of the type of fat consumed, but not in the fasting state.

Our results show that fasting and PP TGRL also increase platelet $\mathrm{TxB}_{2}$ concentration with differences in the extent of TGRL-induced stimulation according to the agonist used. We aimed to use physiological agonists that would not interfere with the lipid composition of TGRL and platelets (eg: arachidonic acid), and that would activate platelets by different signalling pathways. Tested collagen concentrations induce a moderate stimulation while used thrombin concentrations provoke strong platelet activation. The platelets used are from healthy donors, so it is possible that the effect of fasting and PP TGRL would be greater on platelets from T2D patients, since they are already hyperactive in the basal state. 
364 Overall, we acknowledge some limitations to our study. The test fats were comprised in 365 mixed meals so we cannot exclude that other components of the meals could have had an 366 effect on TGRL composition and their effect on platelets, for example compounds from 367 coffee or tea. Also, we could not obtain dietary data from the patients, which would have been 368 useful to evaluate the usual fat intake, but randomization reduces the risk of bias in this case. 369 Finally, our trial included only women, because of their higher risk for cardiovascular morbidity and mortality compared to diabetic men, so our results would need to be confirmed 371 in T2D men.

372 In conclusion, this trial shows that $20 \mathrm{~g}$ of lipids is sufficient to modify the FA composition of 373 TGRL 4 hours after the ingestion in T2D patients. We confirm that fasting TGRL from T2D 374 patients activate platelets and further demonstrate that PP TGRL have a similar enhancing 375 effect on platelet aggregation and $\mathrm{TxB}_{2}$ formation, independently of their FA composition. 376 This study also highlights the importance of TG management in the diabetic population for 377 prevention of cardiovascular complications, as well as dietary counseling regarding fat intake.

378 Our randomized trial shows that a realistic acute intake of either a commercial hazelnut cocoa 379 spread or butter has a similar detrimental effect on TGRL-induced platelet activation 380 compared with TGRL from fasting patients with T2D. 


\section{ACKNOWLEDGEMENTS}

We thank the nurses and the medical team of the department of Endocrinology, Metabolic Disease, Diabetes and Nutrition at Louis Pradel hospital for their essential collaboration and the dieticians at Centre de Recherche en Nutrition Humaine Rhône-Alpes for designing the test breakfasts.

\section{FUNDING}

This work was supported by Inserm and promoted by Hospices Civils de Lyon. MMB received a doctoral contract from the Ministère de l'Enseignement supérieur, de la Recherche et de l'Innovation and a grant from the Nouvelle Société Francophone d'Athérosclérose. CC thanks the CNRS.

\section{CONFLICT OF INTEREST STATTEMENT}

MCM received other research funding on other topics from Sodiaal-Candia R\&D, the Centre National Interprofessionnel de l'Economie Laitière (CNIEL, French Dairy Interbranch Organization) and Nutricia Research and has consultancy activities for food \& dairy companies. These activities had no link with the present study. MCM was the coordinator of a research project funded by the National Research Agency on the valuing and health properties of buttermilk and milk polar lipids (VALOBAB, ANR-2011-0007), in which DC participated; the present study is not part of this project. PM received research grants from Akcea, honoraria for clinical trials or talks paid to his university or to him from Akcea, Amgen, Boehringer, MSD, Novo Nordisk and Sanofi. Other authors have no conflict of interest to declare. 


\section{REFERENCES}

407

408

409

410

411

412

413

414

415

416

417

418

419

420

421

422

423

424

425

426

427

428

429

430

[1] Einarson, T. R., Acs, A., Ludwig, C., Panton, U. H., Prevalence of cardiovascular disease in type 2 diabetes: a systematic literature review of scientific evidence from across the world in 2007-2017. Cardiovasc Diabetol 2018, 17, 83.

[2] Kautzky-Willer, A., Kamyar, M. R., Gerhat, D., Handisurya, A., et al., Sex-specific differences in metabolic control, cardiovascular risk, and interventions in patients with type 2 diabetes mellitus. Gender medicine 2010, 7, 571-583.

[3] Huxley, R., Barzi, F., Woodward, M., Excess risk of fatal coronary heart disease associated with diabetes in men and women: meta-analysis of 37 prospective cohort studies. Bmj 2006, 332, 73-78.

[4] Santilli, F., Simeone, P., Liani, R., Davi, G., Platelets and diabetes mellitus. Prostaglandins Other Lipid Mediat 2015, 120, 28-39.

[5] Taskinen, M. R., Boren, J., New insights into the pathophysiology of dyslipidemia in type 2 diabetes. Atherosclerosis 2015, 239, 483-495.

[6] Boulet, M. M., Cheillan, D., Di Filippo, M., Buisson, C., et al., Large triglyceride-rich lipoproteins from fasting patients with type 2 diabetes activate platelets. Diabetes \& Metabolism 2020, 46, 54-60.

[7] Zilversmit, D. B., Atherogenesis: a postprandial phenomenon. Circulation 1979, 60, 473485.

[8] Iso, H., Naito, Y., Sato, S., Kitamura, A., et al., Serum triglycerides and risk of coronary heart disease among Japanese men and women. American journal of epidemiology 2001, 153, 490-499.

[9] Nordestgaard, B. G., Benn, M., Schnohr, P., Tybjaerg-Hansen, A., Nonfasting triglycerides and risk of myocardial infarction, ischemic heart disease, and death in men and women. Jama 2007, 298, 299-308. 
[10] Freiberg, J. J., Tybjaerg-Hansen, A., Jensen, J. S., Nordestgaard, B. G., Nonfasting triglycerides and risk of ischemic stroke in the general population. Jama 2008, 300, 21422152.

[11] Egeland, G. M., Igland, J., Sulo, G., Nygard, O., et al., Non-fasting triglycerides predict incident acute myocardial infarction among those with favourable HDL-cholesterol: Cohort Norway. European journal of preventive cardiology 2015, 22, 872-881.

[12] Eberly, L. E., Stamler, J., Neaton, J. D., Relation of triglyceride levels, fasting and nonfasting, to fatal and nonfatal coronary heart disease. Archives of internal medicine 2003, $163,1077-1083$.

[13] Bansal, S., Buring, J. E., Rifai, N., Mora, S., et al., Fasting compared with nonfasting triglycerides and risk of cardiovascular events in women. JAMA 2007, 298, 309-316.

[14] Sloop, C. H., Dory, L., Roheim, P. S., Interstitial fluid lipoproteins. J Lipid Res 1987, 28, 225-237.

[15] Nakano, T., Nakajima, K., Niimi, M., Fujita, M. Q., et al., Detection of apolipoproteins B-48 and B-100 carrying particles in lipoprotein fractions extracted from human aortic atherosclerotic plaques in sudden cardiac death cases. Clin Chim Acta 2008, 390, 38-43.

[16] Aviram, M., Brook, J. G., Platelet activation by plasma lipoproteins. Prog Cardiovasc Dis 1987, 30, 61-72.

[17] Englyst, N. A., Taube, J. M., Aitman, T. J., Baglin, T. P., Byrne, C. D., A novel role for CD36 in VLDL-enhanced platelet activation. Diabetes 2003, 52, 1248-1255.

[18] Knofler, R., Nakano, T., Nakajima, K., Takada, Y., Takada, A., Remnant-like lipoproteins stimulate whole blood platelet aggregation in vitro. Thromb Res 1995, 78, 161171. 
454

455

456

457

458

459

460

461

462

463

464

465

466

467

468

469

470

471

472

473

474

475

476

[19] Bysted, A., Hølmer, G., Lund, P., Sandström, B., Tholstrup, T., Effect of dietary fatty acids on the postprandial fatty acid composition of triacylglycerol-rich lipoproteins in healthy male subjects. European Journal of Clinical Nutrition 2005, 59, 24-34.

[20] Guillot, N., Caillet, E., Laville, M., Calzada, C., et al., Increasing intakes of the longchain $\omega-3$ docosahexaenoic acid: effects on platelet functions and redox status in healthy men. The FASEB Journal 2009, 23, 2909-2916.

[21] Skeaff, C. M., Hodson, L., McKenzie, J. E., Dietary-Induced Changes in Fatty Acid Composition of Human Plasma, Platelet, and Erythrocyte Lipids Follow a Similar Time Course. The Journal of Nutrition 2006, 136, 565-569.

[22] Renaud, S., Godsey, F., Dumont, E., Thevenon, C., et al., Influence of long-term diet modification on platelet function and composition in Moselle farmers. The American Journal of Clinical Nutrition 1986, 43, 136-150.

[23] RENAUD, S., DUMONT, E., BAUDIER, F., ORTCHANIAN, E., SYMINGTON, I. S., Effect of smoking and dietary saturated fats on platelet functions in Scottish farmers. Cardiovascular research 1985, 19, 155-159.

[24] Jakubowski, J. A., Ardlie, N. G., Modification of human platelet function by a diet enriched in saturated or polyunsaturated fat. Atherosclerosis 1978, 31, 335-344.

[25] Razmara, M., Hjemdahl, P., Yngen, M., Ostenson, C. G., et al., Food intake enhances thromboxane receptor-mediated platelet activation in type 2 diabetic patients but not in healthy subjects. Diabetes Care 2007, 30, 138-140.

[26] Spectre, G., Östenson, C.-G., Li, N., Hjemdahl, P., Postprandial platelet activation is related to postprandial plasma insulin rather than glucose in patients with type 2 diabetes. Diabetes 2012, 61, 2380-2384. 
477 [27] Folch, J., Lees, M., Sloane Stanley, G. H., A simple method for the isolation and 478 purification of total lipides from animal tissues. The Journal of biological chemistry 1957, $479226,497-509$.

480

481

482

483

484

485

486

487

488

489

490

491

492

493

494

495

496

497

498

499

500

[28] Lowry, O. H., Rosebrough, N. J., Farr, A. L., Randall, R. J., Protein measurement with the Folin phenol reagent. J Biol Chem 1951, 193, 265-275.

[29] Vors, C., Pineau, G., Gabert, L., Drai, J., et al., Modulating absorption and postprandial handling of dietary fatty acids by structuring fat in the meal: a randomized crossover clinical trial. Am J Clin Nutr 2013, 97, 23-36.

[30] Lagarde, M., Bryon, P. A., Guichardant, M., Dechavanne, M., A simple and efficient method for platelet isolation from their plasma. Thromb Res 1980, 17, 581-588.

[31] Born, G. V., Aggregation of blood platelets by adenosine diphosphate and its reversal. Nature 1962, 194, 927-929.

[32] Colas, R., Sassolas, A., Guichardant, M., Cugnet-Anceau, C., et al., LDL from obese patients with the metabolic syndrome show increased lipid peroxidation and activate platelets. Diabetologia 2011, 54, 2931-2940.

[33] Bradford, M. M., A rapid and sensitive method for the quantitation of microgram quantities of protein utilizing the principle of protein-dye binding. Analytical biochemistry $1976,72,248-254$.

[34] Sciarrillo, C. M., Koemel, N. A., Tomko, P. M., Bode, K. B., Emerson, S. R., Postprandial Lipemic Responses to Various Sources of Saturated and Monounsaturated Fat in Adults. Nutrients 2019, 11, 1089.

[35] Dubois, C., Beaumier, G., Juhel, C., Armand, M., et al., Effects of graded amounts (0-50 g) of dietary fat on postprandial lipemia and lipoproteins in normolipidemic adults. Am J Clin Nutr 1998, 67, 31-38. 
501 [36] Mekki, N., Charbonnier, M., Borel, P., Leonardi, J., et al., Butter differs from olive oil

502

503

504

505

506

507

508

509

510

511

512

513

514

515

516

517

518

519

520

521

522

523

524

525

and sunflower oil in its effects on postprandial lipemia and triacylglycerol-rich lipoproteins after single mixed meals in healthy young men. $J$ Nutr 2002, 132, 3642-3649.

[37] Michalski, M. C., Genot, C., Gayet, C., Lopez, C., et al., Multiscale structures of lipids in foods as parameters affecting fatty acid bioavailability and lipid metabolism. Prog Lipid Res 2013, 52, 354-373.

[38] Renaud, S. C., Ruf, J. C., Petithory, D., The positional distribution of fatty acids in palm oil and lard influences their biologic effects in rats. J Nutr 1995, 125, 229-237.

[39] Innis, S. M., Dyer, R. A., Lien, E. L., Formula containing randomized fats with palmitic acid (16:0) in the 2-position increases 16:0 in the 2-position of plasma and chylomicron triglycerides in formula-fed piglets to levels approaching those of piglets fed sow's milk. $J$ Nutr 1997, 127, 1362-1370.

[40] Pufal, D. A., Quinlan, P. T., Salter, A. M., Effect of dietary triacylglycerol structure on lipoprotein metabolism: A comparison of the effects of dioleoylpalmitoylglycerol in which palmitate is esterified to the 2-or 1(3)-position of the glycerol. Biochimica et Biophysica Acta (BBA) - Lipids and Lipid Metabolism 1995, 1258, 41-48.

[41] Karupaiah, T., Sundram, K., Effects of stereospecific positioning of fatty acids in triacylglycerol structures in native and randomized fats: a review of their nutritional implications. Nutrition \& Metabolism 2007, 4, 16.

[42] Aviram, M., Fuhrman, B., Brook, J. G., Postprandial plasma lipoproteins in normal and hypertriglyceridaemic subjects and their in vitro effect on platelet activity: differences between saturated and polyunsaturated fats. Scand J Clin Lab Invest 1986, 46, 571-579.

[43] Panarotto, D., Remillard, P., Bouffard, L., Maheux, P., Insulin resistance affects the regulation of lipoprotein lipase in the postprandial period and in an adipose tissue-specific manner. Eur J Clin Invest 2002, 32, 84-92. 
Boulet et al. 2020, Page 25

526 [44] Delgado-Lista, J., Lopez-Miranda, J., Cortes, B., Perez-Martinez, P., et al., Chronic

527 dietary fat intake modifies the postprandial response of hemostatic markers to a single fatty 528 test meal. Am J Clin Nutr 2008, 87, 317-322.

529

530 
Boulet et al. 2020, Page 26

\section{AUTHOR CONTRIBUTIONS}

532 MMB, CC, DC, MCM and PM designed the study. PM was the principal investigator of the 533 clinical trial. TLB was the clinical research associate assigned to the trial. SLP, JAN and PM 534 collaborated to the writing of the Scientific Ethics Committee application. MMB, CB, CC, 535 and MDF performed the experiments and collected the data. MMB and CC analyzed the data. 536 MMB, CC, DC, MCM and PM interpreted the data. MMB and CC wrote the manuscript. DC, 537 MCM and PM critically revised the manuscript. All co-authors read, commented and 538 approved the manuscript.

539 
TABLE 1. Clinical and biological parameters of the study participants

\begin{tabular}{|c|c|c|c|c|}
\hline Characteristics & $\begin{array}{c}\text { Type } 2 \\
\text { diabetes } \\
(n=30)\end{array}$ & $\begin{array}{c}\text { Type } 2 \text { diabetes } \\
\text { HCS group } \\
(n=15)\end{array}$ & $\begin{array}{l}\text { Type } 2 \text { diabetes } \\
\text { Butter group } \\
(n=15)\end{array}$ & $\mathbf{p}$ \\
\hline Age (years) & $65.5 \pm 1.4$ & $62.9 \pm 2.3$ & $68.1 \pm 1.4$ & $\mathrm{NS}$ \\
\hline $\mathrm{BMI}\left(\mathrm{kg} / \mathrm{m}^{2}\right)$ & $34.1 \pm 1.0$ & $34.4 \pm 1.7$ & $33.9 \pm 1.2$ & NS \\
\hline Duration of diabetes (years) & $12.9^{*} \pm 1.4$ & $12.5 \pm 1.7$ & $13.2 \ddagger \pm 2.4$ & $\mathrm{NS}$ \\
\hline \multicolumn{5}{|l|}{ Glucose homeostasis } \\
\hline $\mathrm{HbA}_{1 \mathrm{c}}(\%)$ & $7.8 \pm 0.2$ & $7.7 \pm 0.3$ & $7.8 \pm 0.3$ & NS \\
\hline Glycemia (mmol/L) & $9.0 \pm 0.7$ & $8.8 \pm 1.0$ & $9.3 \pm 0.9$ & NS \\
\hline Insulin (mU/L) & $12.3^{*} \pm 1.3$ & $11.2 \pm 1.4$ & $13.4 \neq \pm 2.3$ & NS \\
\hline HOMA-IR & $5.0^{*} \pm 0.7$ & $4.1 \pm 0.4$ & $5.9 \neq \pm 1.4$ & NS \\
\hline \multicolumn{5}{|l|}{ Plasma lipids (mmol/L) } \\
\hline Triglycerides & $1.69 \pm 0.1$ & $1.53 \pm 0.1$ & $1.85 \pm 0.3$ & NS \\
\hline Total cholesterol & $4.55 \pm 0.2$ & $4.49 \pm 0.2$ & $4.62 \pm 0.2$ & $\mathrm{NS}$ \\
\hline HDL cholesterol & $1.24 \pm 0.1$ & $1.27 \pm 0.1$ & $1.21 \pm 0.1$ & NS \\
\hline LDL cholesterol & $2.56 \pm 0.1$ & $2.53 \pm 0.2$ & $2.60 \pm 0.2$ & NS \\
\hline
\end{tabular}

Plasma apolipoproteins B (g/L)

Total ApoB

ApoB-100

ApoB-48

$$
0.89 \dagger \pm 0.04
$$

$0.88 \dagger \pm 0.04$

$0.85 \ddagger \pm 0.05$

$0.006 \dagger \pm 0.001$

$0.005 \pm 0.001$

$0.92 \ddagger \pm 0.1$

NS

Antidiabetic and lipid lowering-medication (n(\%))

Metformin

Sulfonylurea
$28(93)$

$18(60)$
$14(93)$

$8(53)$
$0.91 \neq \pm 0.1 \quad \mathrm{NS}$

$0.006 \S \pm 0.001 \quad \mathrm{NS}$ 
Boulet et al. 2020, Page 28

$\begin{array}{lccc}\text { Gliptin } & 8(27) & 4(27) & 4(27) \\ \text { GLP-1 analogs } & 11(37) & 5(33) & 6(40) \\ \text { Acarbose } & 1(3) & 0 & 1(7) \\ \text { Statins } & 18(60) & 8(53) & 10(67) \\ \text { Ezetimibe } & 1(3) & 1(7) & 0\end{array}$

$542 *_{\mathrm{:}=29}+\overline{\mathrm{n}=28}, \overline{\mathrm{n}=14}, \S: \mathrm{n}=13 \overline{\text {. Data are presented as means } \pm \text { SEM. HCS: hazelnut-cocoa spread, NS: not }}$

543 significant. 
544 TABLE 2. Fatty acid composition of the fats in the test breakfasts

\begin{tabular}{lcc}
\hline FA $($ mol \%) & HCS & Butter \\
\hline $\mathbf{1 2 : 0}$ & 0.1 & 4.9 \\
$\mathbf{1 4 : 0}$ & 0.9 & 16.1 \\
$\mathbf{1 5 : 0}$ & 0 & 1.6 \\
$\mathbf{1 6 : 0}$ & 33.2 & 42.2 \\
$\mathbf{1 8 : 0}$ & 6.5 & 10.6 \\
$\mathbf{1 8 : 1}$ n-9 & 45.2 & 15.1 \\
trans 18:1 n-9 & 4.3 & 1.7 \\
$\mathbf{1 8 : 2}$ n-6 & 8.3 & 1.0 \\
$\mathbf{1 8 : 3}$ n-3 & 0.2 & 0.2 \\
$\mathbf{2 0 : 0}$ & 0.3 & 0.1 \\
$\mathbf{2 2 : 0}$ & 0 & 0.1 \\
\hline
\end{tabular}

Total

\begin{tabular}{lcc}
\hline SFA & 41.0 & 78.8 \\
MUFA & 50.5 & 19.9 \\
PUFA & 8.5 & 1.3
\end{tabular}

545 Minor FA are not listed. HCS: hazelnut cocoa spread, FA: fatty acids, SFA: saturated fatty acids 546

547 
549 TABLE 3. Fatty acid composition of TGRL total lipids

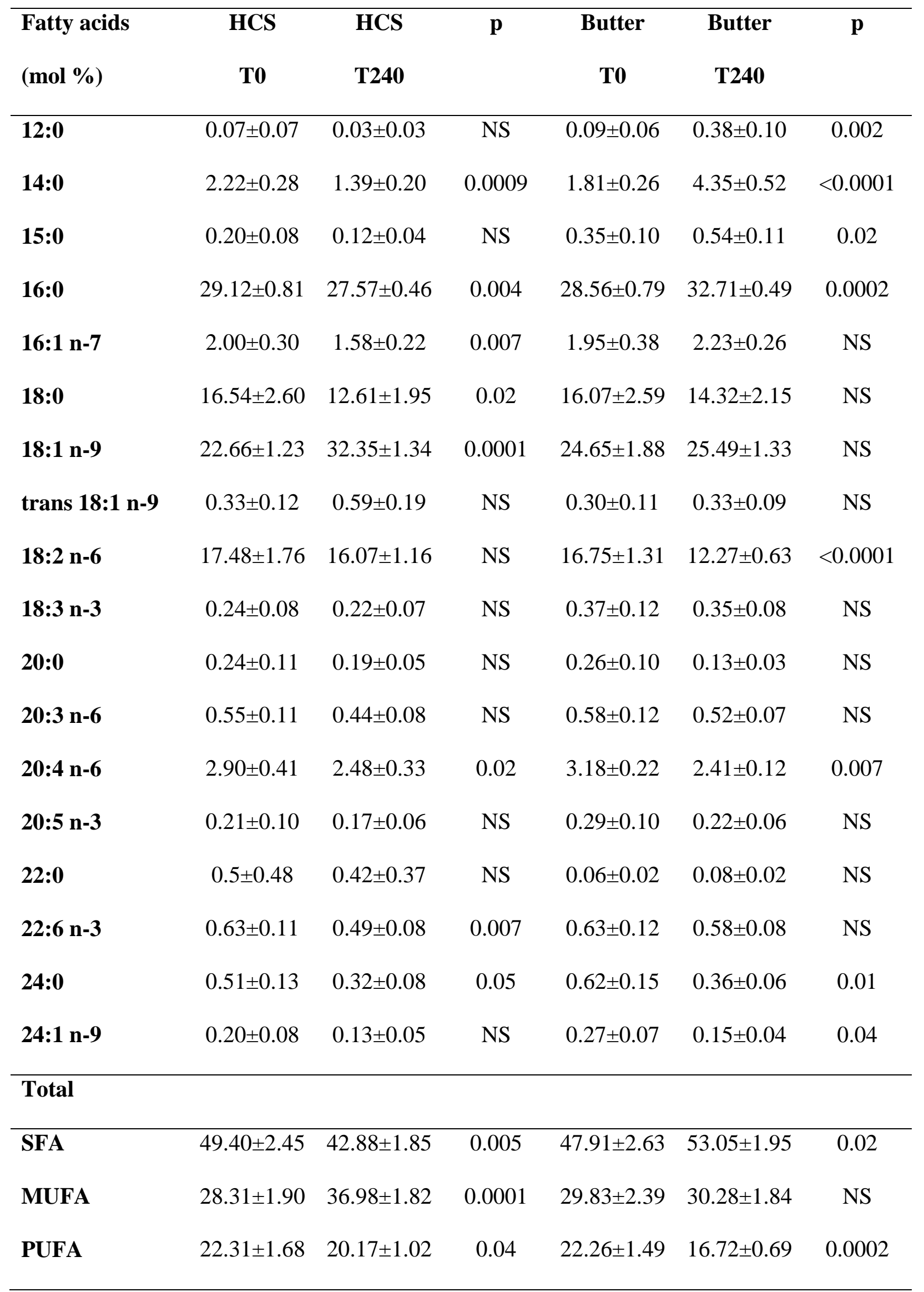


Boulet et al. 2020, Page 31

550 Data are presented as means \pm SEM. Other minor FA are not listed. HCS: hazelnut cocoa spread, NS: not 551 significant, SFA: saturated fatty acids.

552

553 
554

555

556

557

558

559

560

561

562

563

564

565

566

567

568

569

570

571

572

573

574

575

576

577

FIGURE LEGENDS

FIGURE 1. Increase in plasma ApoB-48 and triglyceride concentrations following the test breakfasts

(A) Plasma ApoB-48. (B) Plasma triglycerides. Results are expressed as mean \pm SEM of 15 patients in each group: ${ }^{*} p<0.05$ for both parameters. HCS: hazelnut cocoa spread.

FIGURE 2. Increase of TGRL triglyceride concentration and diameter following the test breakfasts

(A) TGRL triglyceride concentration in plasma. (B) TGRL hydrodynamic diameter. Results are expressed as mean \pm SEM of 15 patients in each group: ${ }^{*} p<0.01$. HCS: hazelnut cocoa spread.

FIGURE 3. Modification of the fatty acid profile of TGRL total lipids following the test breakfasts

Results are expressed as mean of the delta of (FA mol\% at T240-FA mol\% at T0) \pm SEM of 15 patients in each group: ${ }^{*} p<0.05$. HCS: hazelnut cocoa spread, SFA: saturated fatty acids.

FIGURE 4. Collagen-stimulated platelet aggregation is increased by fasting and postprandial TGRL from type 2 diabetic patients

Experiences were performed with platelets from 8 different donors. Results are expressed as mean \pm SEM of aggregation percentage of collagen-stimulated platelets without TGRL (black bars) or pre-incubated with fasting (white bars) or PP TGRL (brown and light blue bars) of 15 patients in each group* $p<0.01$. HCS: hazelnut chocolate spread. 
FIGURE 5. Effects of fasting and postprandial TGRL on agonist-stimulated platelet thromboxane $\mathbf{B}_{2}$

(A) Collagen stimulation. Results are expressed as mean platelet $\mathrm{TxB}_{2}$ concentrations in $\mathrm{pmol} / \mathrm{mg}$ of total proteins $\pm \mathrm{SEM}$ of 15 patients in each group $* p<0.05$. ${ }^{\#} \mathrm{p}=0.07 . \mathrm{HCS}$ : hazelnut cocoa spread.

(B) Thrombin stimulation. Results are expressed as mean platelet $\mathrm{TxB}_{2}$ concentrations in $\mathrm{pmol} / \mathrm{mg}$ of total proteins \pm SEM of 15 patients in each group $* p<0.05$. ${ }^{*} \mathrm{p}=0.06$. HCS: hazelnut cocoa spread.

Experiments were performed with platelets from 8 different donors.

\section{FIGURE 6. Platelet activation by postprandial TGRL is concentration dependant, but}

\section{does not differ according to the fat consumed}

Results are expressed as mean platelet $\mathrm{TxB}_{2}$ concentrations in $\mathrm{pmol} / \mathrm{mg}$ of total proteins \pm SEM. $\mathrm{n}=9, * p<0.05 .{ }^{*} \mathrm{p}=0.06$. HCS: hazelnut cocoa spread, control: platelets + thrombin, TGRLT0_25: platelets $+25 \mu \mathrm{g} / \mathrm{mL}$ TGRL T0 + thrombin, TGRL T240_25: platelets + 25 $\mu \mathrm{g} / \mathrm{mL}$ TGRL T240 + thrombin, TGRL T240_50: platelets + $50 \mu \mathrm{g} / \mathrm{mL}$ TGRL T240 + thrombin. Experiment was performed with platelets from 1 donor. 